\title{
Hallazgos oftalmológicos en recién nacidos de mujeres con infección por el virus Zika durante el embarazo: una serie de casos
}

\section{Ophthalmologic findings in newborns of women with Zika virus infection during pregnancy: A case series}

\author{
Adriana Dávila-Camargo ${ }^{1 *}$, Jaime J. Durán-Nah² y Alfonso Dupinet-Sánchez ${ }^{1}$

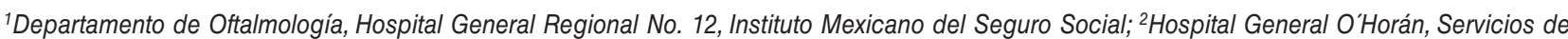
Salud de Yucatán. Mérida, Yuc., México

\section{Resumen}

Objetivo: Describir los hallazgos oculares en recién nacidos (RN) de mujeres con infección por el virus del zika (ZIKV) durante la gestación. Materiales y Métodos: Entre noviembre de 2016 y marzo de 2017, veintiséis RN de mujeres que tuvieron infección por Zika confirmada mediante PCR fueron examinados en el departamento de Oftalmología de un Hospital del Instituto Mexicano del Seguro Social en Mérida, México. Resultados: La edad gestacional en el momento del diagnóstico con ZIKV fue de $29.1 \pm$ 6.9 semanas (intervalo de confianza del 95\% 26.3-31.9). Un caso (3.8\%) fue diagnosticado durante el primer trimestre y 15 casos (57.7\%) durante el tercer trimestre de la gestación. Dieciséis recién nacidos (61.5\%) fueron del sexo masculino. Ningún RN tuvo microcefalia ni anormalidades oculares. Conclusiones: No encontramos microcefalia ni anormalidades oftalmológicas probablemente debido a una exposición antigénica previa a enfermedades por flavivirus como el dengue en la península de Yucatán, a que las infecciones fueron en su mayoría durante el tercer trimestre o debido al corto tiempo que tiene el Zika en Yucatán. No obstante, los oftalmólogos deben estar conscientes de las posibles complicaciones oftalmológicas asociadas con el virus Zika.

Palabras clave: Virus Zika. Mirocefalia. Arbovirus.

\section{Abstract}

Objective: Describe ocular findings in newborns of women with Zika virus (ZIKV) infection during gestation. Materials and Methods: From November 2016 to March 2017, 26 newborns of women with ZIKV infection, confirmed with a reverse transcription polymerase chain reaction test, were examined at the Ophthalmology Department of a General Hospital belonging to the Mexican Institute of Social Security in Merida, Yucatan, Mexico. Results: Maternal gestational age at the time of ZIKV diagnosis averaged $29.1 \pm 6.9$ weeks (95\% confidence interval 26.3-31.9). One case (3.8\%) was diagnosed during the first trimester and 15 cases $(57.7 \%)$ during the third trimester of gestation. 16 newborns (61.5\%) were male. Neither microcephaly nor abnormal ocular abnormalities were found in any newborn. Conclusions: No microcephaly or ocular abnormalities were found in our case series, perhaps due to previous antigenic exposure to flavivirus diseases such as dengue in the Yucatan Peninsula, to infection mostly during the third trimester, or to the short period of time that the ZIKV has been in our country. Nonetheless, ophthalmologists should be aware of the possible ophthalmological complications associated with ZIKV.

Key words: Zika virus infection. Microcephaly. Arboviruses.

\section{Correspondencia:}

*Adriana Dávila-Camargo

Chimalpopoca, 14

Col. Obrera

Fecha de recepción: 30-08-2017

Fecha de aceptación: 22-11-2017

DOI: 10.24875/RMO.M18000035
Disponible en internet: 02-07-2018 Rev Mex Oftalmol. 2018;92(4):187-190

www.rmo.com.mx E-mail: adrydav@gmail.com 0187-4519/@ 2017 Sociedad Mexicana de Oftalmología. Publicado por Permanyer México. Este es un artículo Open Access bajo la licencia CC BY-NC-ND (http://creativecommons.org/licenses/by-nc-nd/4.0/). 


\section{Introducción}

Según los datos epidemiológicos, la primera infección por el virus Zika (ZIKV) en México se identificó en octubre de 2015. En enero de 2017, se confirmaron más casos autóctonos de Zika en al menos 25 estados, y en marzo de 2017, las autoridades sanitarias mexicanas informaron 4390 casos de infección por ZIKV en mujeres embarazadas, siendo el estado de Yucatán el segundo con la tasa de incidencia más alta (642 casos) ${ }^{1}$.

Informes previos sugieren que la infección por ZIKV puede transmitirse verticalmente desde la madre al feto durante el embarazo, habiéndose descrito un síndrome congénito de Zika (SCZ), particularmente cuando la infección ocurre durante el primer trimestre ${ }^{2,3}$. Como parte de sus criterios diagnósticos ${ }^{4}$, el SCZ se caracteriza por microcefalia y anomalías oculares tales como moteado focal de pigmento, atrofia coriorretiniana, hipoplasia del nervio óptico y excavación severa de la papila ${ }^{5}$. En México, se han identificado cinco casos de SCZ desde marzo de $2017^{6}$.

Los datos epidemiológicos de los hallazgos oculares en recién nacidos con $\mathrm{SCZ}$ aún están en investigación ${ }^{7,8}$. En los casos de SCZ de México se documentó microcefalia, pero no se describieron anormalidades oculares $^{5}$. Estudios de Ventura et al. en Brasil describen una asociación probable entre la microcefalia y las anormalidades oculares ${ }^{9}$, pero de Paula Freitas et al., también en Brasil, describieron que hasta el $65 \%$ de los recién nacidos con microcefalia no tenían anomalías oculares ${ }^{10}$. Hasta donde sabemos, no hay datos disponibles sobre las anomalías oculares de los recién nacidos con Zika que no presentaron microcefalia.

Dado que la Península de Yucatán en México se considera una zona endémica del ZIKV que afecta a las mujeres embarazadas, creemos que es valioso y oportuno describir los hallazgos oculares de una serie de casos de recién nacidos de mujeres infectadas con ZIKV.

\section{Materiales y métodos}

Desde noviembre de 2016 hasta marzo de 2017, 26 recién nacidos de madres con infección por ZIKV durante el embarazo confirmada mediante una prueba de reacción en cadena de la polimerasa con transcriptasa inversa (PCR) (Instituto de Diagnóstico y Referencia Epidemiológicos, Ciudad de México) ${ }^{11,12}$, fueron examinados por los mismos oftalmólogos (A.D./A.D.) con un oftalmoscopio binocular 125 indirecto Welch Allyn y un lente $\mathrm{BIO}$ indirecto de $20 \mathrm{D}$ de Volk, en el Departamento de Oftalmología de un Hospital General perteneciente al Instituto Mexicano del Seguro Social en Mérida, Yucatán, México. Como parte de la prueba de detección neonatal, también se realizó el panel TORCH (toxoplasmosis, infección por citomegalovirus, infección por virus de rubéola, infección por sífilis e infección por virus de inmunodeficiencia humana), medición de perímetro cefálico, ultrasonido transfontanelar y pruebas de detección auditiva, todos reportados por los departamentos de pediatría, radiología y otorrinolaringología.

Las variables analizadas fueron sexo, edad gestacional materna en el momento del diagnóstico de infección por ZIKV, días de nacimiento en el momento del examen oftalmológico, anomalías de la cabeza (especialmente microcefalia) y hallazgos del examen oftalmológico. La microcefalia, descrita como una circunferencia cefálica en el momento del nacimiento menor que el $3^{\text {er }}$ percentil para la edad gestacional y el sexo ${ }^{13}$, fue examinada e informada previamente en un examen pediátrico. Se utilizaron estadísticas descriptivas para informar los resultados.

\section{Resultados}

Se examinaron 55 recién nacidos de madres que tenían un diagnóstico probable de infección por ZIKV durante el embarazo, aunque se excluyeron 28 recién nacidos porque la infección materna por ZIKV no fue confirmada mediante PCR. Un caso fue positivo por PCR para el virus del dengue, por lo que también se excluyó del análisis. De los 26 casos restantes de madres con infección por ZIKV confirmada mediante PCR, se examinaron 52 ojos de recién nacidos.

Los síntomas clínicos de ZIKV de las mujeres embarazadas se informaron a una edad gestacional que varió de 12 a 38 semanas, con un promedio de $29.1 \pm$ 6.9 semanas (intervalo de confianza del 95\% 26.331.9). Por trimestre de gestación, se diagnosticó 1 caso (3.8\%) durante el primer trimestre, 10 casos (38.5\%) durante el segundo trimestre y 15 casos $(57.7 \%)$ durante el tercer trimestre. Por género, $16(61.5 \%)$ recién nacidos fueron del sexo masculino. No se informó microcefalia en ningún caso. La edad de los recién nacidos en el momento del examen oftalmológico varió de 2 a 154 días de nacimiento (mediana de 15 días, rangos intercuartiles del 25-75\%, 5.7-36 días). El único hallazgo ocular anormal fue una pequeña hemorragia intrarretiniana peripapilar en un recién nacido varón de 4 días de edad con antecedentes médicos de parto con fórceps, cuya madre tuvo ZIKV confirmado mediante PCR en la semana 30 de gestación. 


\section{Discusión}

De los 26 recién nacidos que se examinaron, no se identificaron anomalías oculares relacionadas con la infección por ZIKV6-8. El caso de hemorragia intrarretiniana que encontramos no se consideró asociado con la infección por ZIKV porque probablemente se debió al procedimiento obstétrico utilizado para el nacimiento. Sin embargo, Miranda et al. $^{5}$ han reportado hemorragias sub-retinianas dispersas por fuera de la mácula, como un sello distintivo asociado a la infección por ZIKV, abriendo la posibilidad de que la hemorragia se debiera a la infección con ZIKV.

Las complicaciones oculares primarias asociadas con la infección por ZIKV no parecen ser específicas de recién nacidos, ya que también se han documentado en adultos ${ }^{14}$. Estudios previos han encontrado que el $34.5 \%$ de los bebés con SCZ tienen anormalidades oftálmicas ${ }^{6}$. Por el contrario, teniendo en cuenta que nuestra serie de casos fue mayor que las descritas anteriormente ${ }^{6-8}$, creemos que es interesante analizar las razones por las que no encontramos ninguna anomalía ocular. Las explicaciones podrían ser varias y estar relacionadas en parte con la presencia o ausencia de factores de riesgo en cada población afectada.

Los factores de riesgo de anormalidades oculares asociadas con la infección por ZIKV parecen incluir a los bebés cuyas madres se infectaron durante el primer trimestre del embarazo y cuando hay microcefalia presente $^{6-8,15}$. La incidencia de niños con anomalías oculares asociadas con la infección por ZIKV puede ser menos frecuente $o$ incluso ausente cuando las mujeres contrajeron ZIKV durante el segundo o el tercer trimestre del embarazo ${ }^{10}$. Por otro lado, también se han informado anomalías oculares en un paciente nacido a término sin microcefalia ${ }^{9}$.

En nuestro estudio, el $96 \%$ de las madres adquirió la infección por ZIKV durante el segundo y tercer trimestre de gestación, un período en el que la organogénesis ya está completa, por lo que los efectos nocivos del ZIKVV ${ }^{16}$ podrían atenuarse 0 estar ausentes, lo que podría explicar la ausencia de anomalías oculares $^{17}$. Lo que es más importante, algunos pacientes, incluyendo los nuestros, pueden tener algún tipo de resistencia inmunológica o protección contra los efectos nocivos que el ZIKV tiene sobre el tejido cerebral y sus estructuras relacionadas, como la retina y los nervios oftálmicos ${ }^{18}$.

Apoyando esta idea, no se descarta un efecto protector de reacciones cruzadas con anticuerpos contra el ZIKV desencadenado por una infección previa por el virus del dengue ${ }^{19}$, ya que nuestras mujeres embarazadas viven en un área endémica de flavivirus como lo es la Península de Yucatán, por lo que las exposiciones anteriores a ese u otro flavivirus podría protegerlas. Finalmente, la ausencia de microcefalia y anormalidades oculares en nuestros casos podría deberse al subregistro de casos, o incluso debido al comportamiento estacional del brote de infección por ZIKV, ya que no ha transcurrido el tiempo suficiente desde el inicio del brote de Zika en la Península de Yucatán como para permitirnos ver la verdadera magnitud del problema.

\section{Conclusiones}

No se encontraron anomalías oculares en recién nacidos remitidos a nuestro servicio como parte de un tamizaje para SCZ. Los factores que pueden explicar nuestros hallazgos incluyen el trimestre de gestación en el momento del diagnóstico, la exposición previa a otros flavivirus, el subregistro y el poco tiempo que el ZIKV ha estado en nuestra región. En entornos de alta transmisión como el estado de Yucatán, México, los oftalmólogos deben tener en cuenta la identificación de complicaciones oftalmológicas congénitas asociadas con el ZIKV.

\section{Agradecimientos}

Agradecemos a las autoridades médicas del Hospital General No. 12 por todas las facilidades otorgadas para llevar a cabo nuestra investigación médica.

\section{Responsabilidades éticas}

Protección de personas y animales. Los autores declaran que para esta investigación no se han realizado experimentos en seres humanos ni en animales.

Confidencialidad de los datos. Los autores declaran que han seguido los protocolos de su centro de trabajo sobre la publicación de datos de pacientes.

\section{Derecho a la privacidad y consentimiento infor-} mado. Los autores declaran que en este artículo no aparecen datos de pacientes.

\section{Financiamiento}

Los autores no recibieron financiamiento específico para este trabajo. 


\section{Conflicto de intereses}

\section{Los autores declaran no tener ningún conflicto de intereses.}

\section{Referencias}

1. OMS. Informe Epidemiológico de Zika. México, Organización Panamericana de la Salud/Organización Mundial de la Salud. Zika-Informe Epidemiológico México. Marzo de 2017. Washington DC: OPS, OMS; 2017. Disponible en: http://www.paho.org/hq/index.php?option=com_docman\&task=doc_view\&gid=35106\&ltemid=270. [Consultado el 15 de julio de 2017].

2. Calvet G, Aguiar RS, Melo ASO, et al. Detection and sequencing of Zika virus from amniotic fluid of fetuses with microcephaly in Brazil: a case study. Lancet Infect Dis. 2016;16:653-60.

3. Oliveira Melo AS, Malinger G, Ximenes R, Szejnfeld PO, Alves Sampaio S, Bispo de Filippis AM. Zika virus intrauterine infection causes fetal brain abnormality and microcephaly: tip of the iceberg? Ultrasound Obstet Gynecol. 2016:47:6-7.

4. Costello A, Dua T, Duran P, et al. Defining the syndrome associated with congenital Zika virus infection. Bull World Health Organ. 2016;94:406-406A

5. Miranda HA 2 ${ }^{\text {nd }}$, Costa MC, Frazão MA, Simão N, Franchischini S, Moshfeghi DM. expanded spectrum of congenital ocular findings in microcephaly with presumed Zika infection. Ophthalmology. 2016;123:1788-94.

6. Secretaría de Salud. Dirección General de Epidemiología. Desglose de casos de Síndrome Congénito Asociado a Zika. Abril; 2017. Available from: http://www.gob.mx/salud/documentos/desglose-de-casos-de-sindrome-congenito-asociado-a-zika. [Consulted 15 July 2017].

7. Ventura CV, Maia M, Ventura BV, et al. Ophthalmological findings in infants with microcephaly and presumable intra-uterus Zika virus infection. Arq Bras Oftalmol. 2016;79:1-3.

8. Yepez JB, Murati FA, Pettito M, et al. Ophthalmic manifestations of congenital zika syndrome in Colombia and Venezuela. JAMA Ophthalmol. 2017;135:440-5.
9. Ventura CV, Maia M, Dias N, Ventura LO, Belfort R Jr. Zika: neurological and ocular findings in infant without microcephaly. Lancet. 2016;387:2502.

10. de Paula Freitas B, de Oliveira Dias JR, Prazeres J, et al. Ocular findings in infants with microcephaly associated with presumed zika virus congenital infection in salvador, Brazil. JAMA Ophthalmol. 2016.

11. Lineamientos Para la Vigilancia Epidemiológica y Diagnóstico Por Laboratorio de Infección por Virus Zika, Secretaría de Salud, Mayo; 2016, Versión 3.0. Available from: https://www.gob.mx/cms/uploads/attachment/file/121722/lineamientos_ve_y_lab_virus_zika.pdf. [Consulted 15 July 2017]

12. Lineamientos Estandarizados Para la Vigilancia Epidemiológica y Diagnóstico por Laboratorio de Infección por Virus Zika. Dirección General de Epidemiología, Secretaria de Salud, México, Diciembre; 2015. Available from: https://www.gob.mx/cms/uploads/attachment/file/207354/lineamientos ve y lab virus zika.pdf. [Consulted 15 July 2017].

13. Congenital Microcephaly Case Definitions. Centers for Disease Control and Prevention, 20 Octobe; 2016. Available from: http://www.cdc.gov/ zika/public-health-partners/microcephaly-case-definitions.html- [Consulted 15 July 2017].

14. Kodati S, Palmore TN, Spellman FA, Cunningham D, Weistrop B, Sen HN. Bilateral posterior uveitis associated with Zika virus infection. Lancet. 2017;389:125-6.

15. Ventura CV, Maia M, Travassos SB, et al. Risk factors associated with the ophthalmoscopic findings identified in infants with presumed Zika virus congenital infection JAMA Ophthalmol. 2016;134:912-8.

16. Retallack H, Di Lullo $E$, Arias $C$, et al. Zika virus cell tropism in the developing human brain and inhibition by azithromycin. Proc Natl Acad Sci USA. 2016;113:14408-13.

17. Cauchemez S, Besnard M, Bompard P, et al. Association between Zika virus and microcephaly in French Polynesia, 2013-2015: a retrospective study. Lancet. 2016;387:2125-32.

18. Li C, Deng YQ, Wang S, et al. 25-Hydroxycholesterol protects host against Zika virus infection and its associated microcephaly in a mouse model. Immunity. 2017;46:446-56.

19. Dejnirattisai W, Supasa P, Wongwiwat W, et al. Dengue cirus sero-cross-reactivity drives antibody-dependent enhancement of infection with zika virus. Nat Immunol. 2016;17:1102-8. 